\title{
Further Investigation on the Relaxed Hybrid Steepest-Descent Methods for Variational Inequalities with $k$-Strict Pseudocontractions
}

\author{
Qian-Fen Gong ${ }^{1}$ and Dao-Jun Wen ${ }^{2}$ \\ ${ }^{1}$ College of Computer Science and Information Engineering, Chongqing Technology and Business University, Chongqing 400067, China \\ ${ }^{2}$ College of Mathematics and Statistics, Chongqing Technology and Business University, Chongqing 400067, China
}

Correspondence should be addressed to Dao-Jun Wen; wendaojun@ctbu.edu.cn

Received 4 January 2014; Accepted 20 February 2014; Published 25 March 2014

Academic Editor: Jian-Wen Peng

Copyright (C) 2014 Q.-F. Gong and D.-J. Wen. This is an open access article distributed under the Creative Commons Attribution License, which permits unrestricted use, distribution, and reproduction in any medium, provided the original work is properly cited.

We modify the relaxed hybrid steepest-descent methods to the case of variational inequality for finding a solution over the set of common fixed points of a finite family of strictly pseudocontractive mappings. The strongly monotone property defined on cost operator was extended to relaxed cocoercive in convergence analysis. Results presented in this paper may be viewed as a refinement and important generalizations of the previously known results announced by many other authors.

\section{Introduction}

Let $H$ be a real Hilbert space with inner product $\langle\cdot, \cdot\rangle$ and norm $\|\cdot\|$, respectively. Let $K$ be a nonempty closed convex subset of $H$ and let $F: H \rightarrow H$ be a nonlinear mapping. We consider the following variational inequality problem: find $q \in K$ such that

$$
\langle F(q), p-q\rangle \geq 0, \quad \forall p \in K,
$$

which was introduced by Stampacchia [1], has emerged as an interesting and fascinating branch of mathematical and engineering sciences. The ideas and techniques of variational inequalities are being applied in structural analysis, economics, optimization, and operations research fields. It has been shown that variational inequalities provide the most natural, direct, simple, and efficient framework for a general treatment of some unrelated problems arising in various fields of pure and applied sciences. In recent years, there have been considerable activities in the development of numerical techniques including projection methods, Wiener-Hopf equations, auxiliary principle, and descent framework for solving variational inequalities; see [2-15] and the references therein.
Recall that a self-mapping $T: H \rightarrow H$ is called a $k$-strict pseudocontraction if there exists a constant $k \in[0,1)$ such that

$$
\begin{aligned}
\|T x-T y\|^{2} \leq & \|x-y\|^{2} \\
& +k\|(I-T) x-(I-T) y\|^{2}, \quad \forall x, y \in H .
\end{aligned}
$$

We use $\operatorname{Fix}(T)$ to denote the fixed point set of $T$; that is, $\operatorname{Fix}(T):=\{x \in H: T x=x\}$. As $k=0, T$ is said to be nonexpansive; that is,

$$
\|T x-T y\| \leq\|x-y\|, \quad \forall x, y \in H .
$$

$T$ is said to be pseudocontractive if $k=1$ and is also said to be strongly pseudocontractive if there exists a positive constant $\lambda \in(0,1)$ such that $T+\lambda I$ is pseudocontractive. Clearly, the class of $k$-strict pseudocontractions falls into the one between classes of nonexpansive mappings and pseudocontractions. We remark also that the class of strongly pseudocontractive mappings is independent of the class of $k$ strict pseudocontractions (see, e.g., $[16,17]$ ). 
It is well known that a variational inequality in a smooth Banach space is equivalent to a fixed-point equation containing a sunny nonexpansive retraction from any point of the space onto the feasible set, assumed usually to be closed and convex. However, the sunny nonexpansive retraction is not easy to compute, due to the complexity of the feasible set [18]. In order to overcome this drawback in a Hilbert space, where the retraction is a metric projection, $\mathrm{Xu}$ [19] assumed that the feasible set was the set of common fixed points of a finite family of nonexpansive mappings and introduced a hybrid steepest-descent method. To be more precise, $\mathrm{Xu}$ proposed the following steepest-descent method cyclically in combination with each nonexpansive mapping of a finite family:

$$
u_{k+1}=\lambda_{k+1} u+\left(I-\lambda_{k+1} A\right) T_{[k+1]} u_{k},
$$

where $T_{[n]}=T_{n \bmod N}, A$ is a self-adjoint, linear, bounded, and strongly positive mapping, $u$ is some fixed point, and $I$ denotes the identity operator of $H$, and proved that, under the following conditions:

(C1) $\lim _{k \rightarrow \infty} \lambda_{k}=0$;

(C2) $\sum_{k=1}^{\infty} \lambda_{k}=\infty$;

(C3) $\sum_{k=1}^{\infty}\left|\lambda_{k}-\lambda_{k+N}\right|<\infty$ or $\lim _{k \rightarrow \infty}\left(\lambda_{k}-\lambda_{k+N}\right) /$ $\lambda_{k+N}=0$.

Assume, in addition, that

(C4) $K=\operatorname{Fix}\left(T_{1} T_{2} \cdots T_{N-1} T_{N}\right)=\operatorname{Fix}\left(T_{N} T_{1} \cdots\right.$ $\left.T_{N-2} T_{N-1}\right)=\cdots=\operatorname{Fix}\left(T_{2} T_{3} \cdots T_{N} T_{1}\right)$ holds.

Then the sequence $\left\{u_{n}\right\}$, generated by (4), converges strongly to the unique solution of variational inequality (1) with $F(x)=A x-u$.

Recently, Zeng et al. [11] proposed a hybrid steepestdescent method with variable parameters for variational inequalities, and Yao et al. [12] analyzed the strong convergence of three-step relaxed hybrid steepest-descent methods for variational inequalities. Very recently, Liu and Cui [20] showed that the condition

$$
K=\bigcap_{i=1}^{N} \operatorname{Fix}\left(T_{i}\right)=\operatorname{Fix}\left(T_{1} T_{2} \cdots T_{N}\right)
$$

is sufficient for $(\mathrm{C} 4)$ as $K \neq \emptyset$.

In 2011, Buong and Duong [13] proposed an explicit iterative algorithm for a class of variational inequalities. To be more precise, they proved the following theorem.

Theorem BD. Let $H$ be a real Hilbert space and $F: H \rightarrow H$ be a map such that, for some positive constants $L$ and $r, F$ is $L$ Lipschitz continuous and r-strongly monotone. Let $\left\{T_{i}\right\}_{i=1}^{N}$ be $N$ nonexpansive self-maps of $H$ such that $K=\bigcap_{i=1}^{N} \operatorname{Fix}\left(T_{i}\right) \neq \phi$. Then the sequence $\left\{x_{n}\right\}$ defined by

$$
\begin{gathered}
x_{0} \in H, \quad y_{0}^{0}=x_{0}, \\
y_{k}^{i}=\left(1-\beta_{k}^{i}\right) y_{k}^{i-1}+\beta_{k}^{i} T_{i} y_{k}^{i-1}, \quad i=1,2, \ldots, N, \\
x_{k+1}=\left(1-\beta_{k}^{0}\right) x_{k}+\beta_{k}^{0}\left(I-\lambda_{k} \mu F\right) y_{n}, \quad k \geq 0,
\end{gathered}
$$

where the parameters $\lambda_{k} \in(0,1)$ for $k \geq 0, \beta_{k}^{i} \in[\alpha, \beta] \subset$ $(0,1), i=0,1, \ldots, N$, and conditions (C1), (C2), and (C5) $\lim _{k \rightarrow \infty}\left|\beta_{k+1}^{i}-\beta_{k}^{i}\right|=0, i=1,2, \ldots, N$ are satisfied, converges strongly to the unique solution $q \in K$ of variational inequality (1).

It is worth mentioning that almost all the results regarding the existence and convergence of the steepest-descent methods for variational inequality requires that the underlying operator $F$ must be strongly monotone and Lipschitzian continuous. These strict conditions rule out many applications of these methods and their various modifications. This fact motivates to develop other methods or modify the steepestdescent methods with more weaker or general conditions.

In this paper, inspired and motivated by research going on in this area, we introduce a new parallel relaxed hybrid steepest-descent method in combination with a finite family of strict pseudocontractive mappings, which is defined in the following way:

$$
\begin{aligned}
y_{n} & =\left(1-\beta_{n}\right) x_{n}+\beta_{n} \sum_{i=1}^{N} \eta_{i}^{(n)} T_{i} x_{n}, \\
x_{n+1} & =\left(1-\alpha_{n}\right) x_{n}+\alpha_{n}\left(I-\lambda_{n} \mu F\right) y_{n}, \quad n \geq 0,
\end{aligned}
$$

where $\left\{T_{i}\right\}_{i=i}^{N}: H \rightarrow H$ is a finite family of $k_{i}$-strict pseudocontractions, $\left\{\alpha_{n}\right\},\left\{\beta_{n}\right\}$, and $\left\{\eta_{i}^{(n)}\right\}_{i=1}^{N}$ are some positive sequences in $(0,1)$.

Our purpose is not only to extend the relaxed hybrid steepest-descent methods to the case of variational inequality in combination with a finite family of $k_{i}$-strictly pseudocontractive mappings, but also to remove conditions (C3), (C4), and (C5) $\lim _{k \rightarrow \infty}\left|\beta_{k+1}^{i}-\beta_{k}^{i}\right|=0, i=1,2, \ldots, N$ in convergence analysis. Moreover, the strongly monotone property defined on cost operator $F$ was extended to relaxed $(\gamma, r)$-cocoercive. Our results presented in this paper improve and extend the corresponding ones of [5, 11-13, 19-21].

\section{Preliminaries}

Let $K$ be a nonempty closed convex subset of real Hilbert $H$. We use $\rightarrow$ and $\rightarrow$ to denote strong and weak convergence of sequences in $H$, respectively. In order to prove main results, we need the following concepts and results.

Definition 1. A mapping $F: H \rightarrow H$ is called $L$-Lipschitzian continuous if there exists constant $L>0$ such that

$$
\|F(x)-F(y)\| \leq L\|x-y\|, \quad \forall x, y \in H .
$$

Definition 2. A mapping $F: H \rightarrow H$ is called

(i) $r$-strongly monotone if there exists a constant $r>0$ such that

$$
\langle F(x)-F(y), x-y\rangle \geq r\|x-y\|^{2}, \quad \forall x, y \in H ;
$$

(ii) $\alpha$-inverse strongly monotonic if there exists a constant $\alpha>0$ such that

$$
\langle F(x)-F(y), x-y\rangle \geq \alpha\|F(x)-F(y)\|^{2}, \quad \forall x, y \in H ;
$$


(iii) relaxed $(\gamma, r)$-cocoercive if there exist constants $\gamma>0$ and $r>0$ such that

$$
\begin{aligned}
\langle F(x)-F(y), x-y\rangle \geq & -\gamma\|F(x)-F(y)\|^{2} \\
& +r\|x-y\|^{2}, \quad \forall x, y \in H .
\end{aligned}
$$

Remark 3. Obviously, a $r$-strongly monotonic mapping must be a relaxed $(\gamma, r)$-cocoercive mapping whenever $\gamma=0$, but the converse is not true. Therefore the class of the relaxed $(\gamma, r)$-cocoercive mappings is a more general class than $r$ strongly monotone.

Lemma 4 (see [22]). Let $H$ be a real Hilbert space, for any fixed $t \in[0,1]$; then

(i) $\|x+y\|^{2} \leq\|x\|^{2}+2\langle y, x+y\rangle$, for all $x, y \in H$;

(ii) $\|t x+(1-t) y\|^{2} \leq t\|x\|^{2}+(1-t)\|y\|^{2}-t(1-t)\|x-y\|^{2}$, for all $x, y \in H$.

Lemma 5 (see [21]). Let $T: H \rightarrow H$ be a $k$-strict pseudocontraction. For $\lambda \in[k, 1)$, define $S: H \rightarrow H$ by $S x=\lambda x+(1-\lambda) T x$ for each $x \in K$. Then $S$ is a nonexpansive mapping such that $\operatorname{Fix}(S)=\operatorname{Fix}(T)$.

Lemma 6 (see [23]). Let $\left\{x_{n}\right\}$ and $\left\{y_{n}\right\}$ be two bounded sequences in a Banach space E such that $x_{n+1}=\left(1-\alpha_{n}\right) x_{n}+$ $\alpha_{n} y_{n}$ for all integers $n \geq 0$. Let $\left\{\alpha_{n}\right\}$ be a sequence in $[0,1]$ with $0<\liminf _{n \rightarrow \infty} \alpha_{n} \leq \limsup _{n \rightarrow \infty} \alpha_{n}<1$. Suppose that

$$
\limsup _{n \rightarrow \infty}\left(\left\|y_{n+1}-y_{n}\right\|-\left\|x_{n+1}-x_{n}\right\|\right) \leq 0 \text {. }
$$

Then $\lim _{n \rightarrow \infty}\left\|x_{n}-y_{n}\right\|=0$.

Lemma 7 (demiclosed principle [24]). Let $K$ be a nonempty closed convex subset of a real Hilbert space $H$ and let $T$ : $K \rightarrow K$ be nonexpansive mapping. Then the mapping $I-T$ is demiclosed; that is, $x_{n} \rightarrow x \in K$ and $(I-T) x_{n} \rightarrow y$ implies $(I-T) x=y$.

Lemma 8 (see [19]). Assume $\left\{a_{n}\right\}$ is a sequence of nonnegative real numbers such that

$$
a_{n+1} \leq\left(1-\gamma_{n}\right) a_{n}+\gamma_{n} \delta_{n}, \quad n \geq 0,
$$

where $\left\{\gamma_{n}\right\}$ is a sequence in $(0,1)$ and $\left\{\delta_{n}\right\}$ is a real sequence such that

(i) $\sum_{n=1}^{\infty} \gamma_{n}=\infty$;

(ii) $\lim \sup _{n \rightarrow \infty} \delta_{n} \leq 0$ or $\sum_{n=1}^{\infty}\left|\gamma_{n} \delta_{n}\right|<\infty$.

Then $\lim _{n \rightarrow \infty} a_{n}=0$.

Proposition 9 (see Acedo and Xu [25]). Let $K$ be a nonempty closed convex subset of a Hilbert space $H$. Given an integer $N \geq 1$, assume that $\left\{T_{i}\right\}_{i=1}^{N}: H \rightarrow H$ is a finite family of $k_{i}$-strict pseudocontractions. Suppose that $\left\{\lambda_{i}\right\}_{i=1}^{N}$ is a positive sequence such that $\sum_{i=1}^{N} \lambda_{i}=1$. Then $\sum_{i=1}^{N} \lambda_{i} T_{i}$ is a $k$-strict pseudocontraction with $k=\max \left\{k_{i}: 1 \leq i \leq N\right\}$.
Proposition 10 (see Acedo and $\mathrm{Xu}$ [25]). Let $\left\{T_{i}\right\}_{i=1}^{N}$ and $\left\{\lambda_{i}\right\}_{i=1}^{N}$ be given as in Proposition 9. Then $\operatorname{Fix}\left(\sum_{i=1}^{N} \lambda_{i} T_{i}\right)=$ $\bigcap_{i=1}^{N} \operatorname{Fix}\left(T_{i}\right)$.

\section{Main Results}

Lemma 11. Let $H$ be a real Hilbert space and let $F: H \rightarrow$ $H$ be a relaxed $(\gamma, r)$-cocoercive and $L$-Lipschitzian continuous mapping. For $\lambda \in(0,1)$ and $0<\mu<2\left(r-\gamma L^{2}\right) / L^{2}$, we have

$$
\left\|T_{\lambda}^{\mu} x-T_{\lambda}^{\mu} y\right\| \leq(1-\lambda \tau)\|x-y\|, \quad \forall x, y \in H,
$$

where $T_{\lambda}^{\mu}:=I-\lambda \mu F$ and $\tau=1-\sqrt{1-\mu\left[2\left(r-\gamma L^{2}\right)-\mu L^{2}\right]} \epsilon$ $(0,1)$.

Proof. By the properties defined on $F$, we obtain

$$
\begin{aligned}
\left\|T_{\lambda}^{\mu} x-T_{\lambda}^{\mu} y\right\|^{2}= & \|x-y-\lambda \mu[F(x)-F(y)]\|^{2} \\
= & \|x-y\|^{2}-2 \lambda \mu\langle F(x)-F(y), x-y\rangle \\
& +\lambda^{2} \mu^{2}\|F(x)-F(y)\|^{2} \\
\leq & \|x-y\|^{2}-2 \lambda \mu \\
& \times\left[-\gamma\|F(x)-F(y)\|^{2}+r\|x-y\|^{2}\right] \\
& +\lambda^{2} \mu^{2}\|F(x)-F(y)\|^{2} \\
\leq & \left(1-2 \lambda \mu r+2 \lambda \mu \gamma L^{2}+\lambda^{2} \mu^{2} L^{2}\right)\|x-y\|^{2} \\
\leq & (1-\lambda \tau)^{2}\|x-y\|^{2},
\end{aligned}
$$

where $\tau=1-\sqrt{1-\mu\left[2\left(r-\gamma L^{2}\right)-\mu L^{2}\right]}$. From $0<\mu<2(r-$ $\left.\gamma L^{2}\right) / L^{2}$, it is easy to obtain that $\tau \in(0,1)$. We immediately conclude the desired results. This completes the proof.

Theorem 12. Let $H$ be a real Hilbert space and let $F: H \rightarrow H$ be a relaxed $(\gamma, r)$-cocoercive and $L$-Lipschitzian continuous mapping. Let $\left\{T_{i}\right\}_{i=1}^{N}: H \rightarrow H$ be a finite family of $k_{i}$-strict pseudocontractions such that $K=\bigcap_{i=1}^{N} \operatorname{Fix}\left(T_{i}\right) \neq \phi$. Suppose $\left\{\eta_{i}^{(n)}\right\}_{i=1}^{N}$ are finite sequences of positive numbers such that $\sum_{i=1}^{N} \eta_{i}^{(n)}=1$ for all $n \geq 0$ and $\lambda_{n} \in(0,1), 0<\mu<$ $2\left(r-\gamma L^{2}\right) / L^{2}$. In addition, for a given point $x_{0} \in H$, (C1), (C2), and the following control conditions are satisfied:

(i) $0<\liminf _{n \rightarrow \infty} \alpha_{n} \leq \limsup _{n \rightarrow \infty} \alpha_{n}<1$;

(ii) $\beta_{n} \in(0,1-k]$ and $\lim _{n \rightarrow \infty} \beta_{n}=\lambda$, where $k=\max \left\{k_{i}\right.$ : $1 \leq i \leq N\}$;

(iii) $\lim _{n \rightarrow \infty}\left|\eta_{i}^{(n+1)}-\eta_{i}^{(n)}\right|=0$.

Then the sequence $\left\{x_{n}\right\}$ generated by (7) converges strongly to the unique element $q \in K$ of the variational inequality (1).

Proof. Putting $W_{n}=\sum_{i=1}^{N} \eta_{i}^{(n)} T_{i}$, we have that $W_{n}: H \rightarrow H$ is a $k$-strict pseudocontraction and $\operatorname{Fix}\left(W_{n}\right)=\bigcap_{i=1}^{N} \operatorname{Fix}\left(T_{i}\right)$ by Propositions 9 and 10, where $k=\max \left\{k_{i}: 1 \leq i \leq N\right\}$. 
First, we show that $S_{n}=\left(1-\beta_{n}\right) I+\beta_{n} W_{n}$ is nonexpansive. Indeed, for each $x, y \in H$, we have

$$
\begin{aligned}
\| S_{n} x & -S_{n} y \|^{2} \\
= & \left\|\left(1-\beta_{n}\right)(x-y)+\beta_{n}\left(W_{n} x-W_{n} y\right)\right\|^{2} \\
= & \left(1-\beta_{n}\right)\|x-y\|^{2}+\beta_{n}\left\|W_{n} x-W_{n} y\right\|^{2} \\
& -\beta_{n}\left(1-\beta_{n}\right)\left\|x-W_{n} x-\left(y-W_{n} y\right)\right\|^{2} \\
\leq & \left(1-\beta_{n}\right)\|x-y\|^{2}+\beta_{n} \\
& \times\left[\|x-y\|^{2}+k\left\|\left(I-W_{n}\right) x-\left(I-W_{n}\right) y\right\|^{2}\right] \\
& -\beta_{n}\left(1-\beta_{n}\right)\left\|\left(I-W_{n}\right) x-\left(I-W_{n}\right) y\right\|^{2} \\
= & \|x-y\|^{2}-\beta_{n}\left(1-\beta_{n}-k\right) \\
& \times\left\|\left(I-W_{n}\right) x-\left(I-W_{n}\right) y\right\|^{2} \\
\leq & \|x-y\|^{2} .
\end{aligned}
$$

It follows from that that the mapping $S_{n}$ is nonexpansive. By Lemma 5, we see that

$$
\operatorname{Fix}\left(S_{n}\right)=\operatorname{Fix}\left(W_{n}\right)=\bigcap_{i=1}^{N} \operatorname{Fix}\left(T_{i}\right) .
$$

Note that $y_{n}=S_{n} x_{n}$ and $p=S_{n} p$ for each $n \geq 1$ as $p \in K$. From (7) and Lemma 11, we obtain

$$
\begin{aligned}
&\left\|x_{n+1}-p\right\| \\
&=\left\|\left(1-\alpha_{n}\right) x_{n}+\alpha_{n}\left(I-\lambda_{n} \mu F\right) y_{n}-p\right\| \\
& \leq\left(1-\alpha_{n}\right)\left\|x_{n}-p\right\| \\
&+\alpha_{n}\left\|\left(I-\lambda_{n} \mu F\right)\left(y_{n}-p\right)-\lambda_{n} \mu F(p)\right\| \\
& \leq\left(1-\alpha_{n}\right)\left\|x_{n}-p\right\|+\alpha_{n}\left\|T_{\lambda_{n}}^{\mu} y_{n}-T_{\lambda_{n}}^{\mu} p\right\| \\
&+\alpha_{n} \lambda_{n} \mu\|F(p)\| \\
& \leq\left(1-\alpha_{n}\right)\left\|x_{n}-p\right\|+\alpha_{n}\left(1-\lambda_{n} \tau\right) \\
& \times\left\|S_{n} x_{n}-p\right\|+\alpha_{n} \lambda_{n} \mu\|F(p)\| \\
& \leq\left(1-\alpha_{n} \lambda_{n} \tau\right)\left\|x_{n}-p\right\|+\alpha_{n} \lambda_{n} \mu\|F(p)\|,
\end{aligned}
$$

where $T_{\lambda_{n}}^{\mu}:=I-\lambda_{n} \mu F$. It follows from induction that

$$
\left\|x_{n}-p\right\| \leq \max \left\{\left\|x_{0}-p\right\|, \frac{\mu}{\tau}\|F(p)\|\right\}, \quad n \geq 1,
$$

which shows that sequence $\left\{x_{n}\right\}$ is bounded and so are $\left\{y_{n}\right\}$ and $\left\{F\left(y_{n}\right)\right\}$.
Next, put $z_{n}=\left(I-\lambda_{n} \mu F\right) y_{n}$. Then, from (7) and $y_{n}=$ $S_{n} x_{n}$, we have that

$$
\begin{aligned}
x_{n+1}= & \left(1-\alpha_{n}\right) x_{n}+\alpha_{n} z_{n}, \\
\left\|z_{n+1}-z_{n}\right\|= & \left\|\left(I-\lambda_{n+1} \mu F\right) y_{n+1}-\left(I-\lambda_{n} \mu F\right) y_{n}\right\| \\
\leq & \left\|y_{n+1}-y_{n}\right\| \\
& +\left\|\lambda_{n+1} \mu F\left(y_{n+1}\right)-\lambda_{n} \mu F\left(y_{n}\right)\right\| \\
\leq & \left\|y_{n+1}-y_{n}\right\| \\
& +\lambda_{n+1} \mu\left\|F\left(y_{n+1}\right)\right\|+\lambda_{n} \mu\left\|F\left(y_{n}\right)\right\| \\
\leq & \left\|S_{n+1} x_{n+1}-S_{n+1} x_{n}\right\| \\
& +\left\|S_{n+1} x_{n}-S_{n} x_{n}\right\|+\left(\lambda_{n+1}+\lambda_{n}\right) \mu M_{1} \\
\leq & \left\|x_{n+1}-x_{n}\right\|+\left\|S_{n+1} x_{n}-S_{n} x_{n}\right\| \\
& +\left(\lambda_{n+1}+\lambda_{n}\right) \mu M_{1},
\end{aligned}
$$

where $M_{1}=\sup _{n \geq 1}\left\{\left\|F\left(y_{n}\right)\right\|\right\}$. Moreover, we note that

$$
\begin{aligned}
\left\|S_{n+1} x_{n}-S_{n} x_{n}\right\| \\
=\left\|\left(1-\beta_{n+1}\right) x_{n}+\beta_{n+1} W_{n+1} x_{n}-\left(1-\beta_{n}\right) x_{n}-\beta_{n} W_{n} x_{n}\right\| \\
=\|\left(\beta_{n}-\beta_{n+1}\right) x_{n}+\left(\beta_{n+1}-\beta_{n}\right) W_{n+1} x_{n} \\
\quad+\beta_{n}\left(W_{n+1} x_{n}-W_{n} x_{n}\right) \| \\
\leq\left|\beta_{n+1}-\beta_{n}\right|\left\|x_{n}-W_{n+1} x_{n}\right\|+\beta_{n}\left\|W_{n+1} x_{n}-W_{n} x_{n}\right\| \\
\leq\left|\beta_{n+1}-\beta_{n}\right|\left\|x_{n}-W_{n+1} x_{n}\right\| \\
\quad+\sum_{i=1}^{N}\left|\eta_{i}^{(n+1)}-\eta_{i}^{(n)}\right|\left\|T_{i} x_{n}\right\| \\
\leq\left|\beta_{n+1}-\beta_{n}\right| M_{2}+\sum_{i=1}^{N}\left|\eta_{i}^{(n+1)}-\eta_{i}^{(n)}\right| M_{2},
\end{aligned}
$$

where $M_{2}=\sup _{n \geq 1}\left\{\left\|T_{i} x_{n}\right\|,\left\|x_{n}-W_{n+1} x_{n}\right\|\right\}$. Combining (21) and (22), we obtain

$$
\begin{aligned}
\| z_{n+1} & -z_{n}\|-\| x_{n+1}-x_{n} \| \\
& \leq\left(\lambda_{n+1}+\lambda_{n}\right) \mu M_{1}+\left|\beta_{n+1}-\beta_{n}\right| M_{2}+\sum_{i=1}^{N}\left|\eta_{i}^{(n+1)}-\eta_{i}^{(n)}\right| M_{2} .
\end{aligned}
$$

By (C1) and conditions (ii)-(iii), we have that

$$
\limsup _{n \rightarrow \infty}\left(\left\|z_{n+1}-z_{n}\right\|-\left\|x_{n+1}-x_{n}\right\|\right) \leq 0 .
$$

It follows from (i) and Lemma 6 that

$$
\lim _{n \rightarrow \infty}\left\|x_{n}-z_{n}\right\|=0 \text {. }
$$


From (20), we have that $x_{n+1}-x_{n}=\alpha_{n}\left(z_{n}-x_{n}\right)$ and

$$
\lim _{n \rightarrow \infty}\left\|x_{n+1}-x_{n}\right\|=0
$$

On the other hand, we note that

$$
\begin{aligned}
& \left\|x_{n}-S_{n} x_{n}\right\| \\
& \leq\left\|x_{n}-x_{n+1}\right\|+\left\|x_{n+1}-S_{n} x_{n}\right\| \\
& =\left\|x_{n}-x_{n+1}\right\|+\left\|\left(1-\alpha_{n}\right) x_{n}+\alpha_{n}\left(I-\lambda_{n} \mu F\right) y_{n}-S_{n} x_{n}\right\| \\
& \leq\left\|x_{n}-x_{n+1}\right\|+\left(1-\alpha_{n}\right)\left\|x_{n}-S_{n} x_{n}\right\| \\
& \quad+\alpha_{n} \lambda_{n} \mu\left\|F\left(y_{n}\right)\right\|,
\end{aligned}
$$

which implies that

$$
\alpha_{n}\left\|x_{n}-S_{n} x_{n}\right\| \leq\left\|x_{n}-x_{n+1}\right\|+\alpha_{n} \lambda_{n} \mu\left\|F\left(y_{n}\right)\right\| .
$$

This together with (i), (C1), and (26), we obtain

$$
\lim _{n \rightarrow \infty}\left\|x_{n}-S_{n} x_{n}\right\|=0
$$

that is,

$$
\lim _{n \rightarrow \infty}\left\|x_{n}-y_{n}\right\|=0
$$

Furthermore, we observe that

$$
\left\|x_{n}-S_{n} x_{n}\right\|=\beta_{n}\left\|x_{n}-W_{n} x_{n}\right\|
$$

It follows from condition (ii) that

$$
\lim _{n \rightarrow \infty}\left\|x_{n}-W_{n} x_{n}\right\|=0
$$

By condition (iii), we may assume that $\eta_{i}^{(n)} \rightarrow \eta_{i}$ as $n \rightarrow \infty$ for every $1 \leq i \leq N$. It is easily seen that each $\eta_{i}>0$ and $\sum_{i=1}^{N} \eta_{i}=1$. Define $W=\sum_{i=1}^{N} \eta_{i} T_{i}$; then $W: K \rightarrow H$ is a $k$-strict pseudocontraction such that $\operatorname{Fix}(W)=\operatorname{Fix}\left(W_{n}\right)=$ $\bigcap_{i=1}^{N} \operatorname{Fix}\left(T_{i}\right)$ by Propositions 9 and 10. Consequently,

$$
\begin{aligned}
\left\|x_{n}-W x_{n}\right\| & \leq\left\|x_{n}-W_{n} x_{n}\right\|+\left\|W_{n} x_{n}-W x_{n}\right\| \\
& \leq\left\|x_{n}-W_{n} x_{n}\right\|+\sum_{i=1}^{N}\left|\eta_{i}^{(n)}-\eta_{i}\right|\left\|T_{i} x_{n}\right\|,
\end{aligned}
$$

which implies that

$$
\lim _{n \rightarrow \infty}\left\|x_{n}-W x_{n}\right\|=0
$$

Combining (32) and (34), we obtain

$$
\lim _{n \rightarrow \infty}\left\|W_{n} x_{n}-W x_{n}\right\|=0
$$

Define $S: K \rightarrow H$ by $S x=(1-\lambda) x+\lambda W x$. By condition (ii) again, we have $\lim _{n \rightarrow \infty} \beta_{n}=\lambda \in(0,1-k]$. Then, $S$ is nonexpansive with $\operatorname{Fix}(S)=\operatorname{Fix}(W)$ by Lemma 5 . Notice that

$$
\begin{aligned}
\| x_{n}- & S x_{n} \| \\
\leq & \left\|x_{n}-S_{n} x_{n}\right\|+\left\|S_{n} x_{n}-S x_{n}\right\| \\
= & \left\|x_{n}-S_{n} x_{n}\right\| \\
& +\left\|\left(1-\beta_{n}\right) x_{n}+\beta_{n} W_{n} x_{n}-(1-\lambda) x_{n}-\lambda W x_{n}\right\| \\
\leq & \left\|x_{n}-S_{n} x_{n}\right\| \\
& +\left|\beta_{n}-\lambda\right|\left\|x_{n}-W x_{n}\right\|+\beta_{n}\left\|W_{n} x_{n}-W x_{n}\right\| .
\end{aligned}
$$

It follows from (29), (34), and (35) that

$$
\lim _{n \rightarrow \infty}\left\|x_{n}-S x_{n}\right\|=0 .
$$

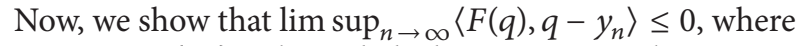
$q \in K$. Since $\left\{x_{n}\right\}$ is bounded, there exists a subsequence $\left\{x_{n_{j}}\right\}$ of $\left\{x_{n}\right\}$. Without loss of generality, we suppose that the sequence $\left\{x_{n_{j}}\right\}$ converges weakly to $\widetilde{q}$ such that

$$
\limsup _{n \rightarrow \infty}\left\langle F(q), q-x_{n}\right\rangle=\lim _{j \rightarrow \infty}\left\langle F(q), q-x_{n_{j}}\right\rangle .
$$

It follows from (37) and Lemma 7 that $\tilde{q} \in K=\operatorname{Fix}(S)=$ $\operatorname{Fix}\left(W_{n}\right)=\bigcap_{i=1}^{N} \operatorname{Fix}\left(T_{i}\right)$. Consequently, by (1), it implies that

$$
\lim _{j \rightarrow \infty}\left\langle F(q), q-x_{n_{j}}\right\rangle \leq 0
$$

Similarly, by (30), there exists a subsequence $\left\{y_{n_{j}}\right\}$ of $\left\{y_{n}\right\}$ that converges weakly to $\tilde{q}$ such that

$$
\limsup _{n \rightarrow \infty}\left\langle F(q), q-y_{n}\right\rangle=\lim _{j \rightarrow \infty}\left\langle F(q), q-y_{n_{j}}\right\rangle \leq 0 .
$$

Finally, we prove that $x_{n} \rightarrow q$ as $n \rightarrow \infty$ as follows. From (7), Lemmas 4 and 11 again, we have

$$
\begin{aligned}
& \left\|x_{n+1}-q\right\|^{2} \\
& =\left\|\left(1-\alpha_{n}\right) x_{n}+\alpha_{n}\left(I-\lambda_{n} \mu F\right) y_{n}-q\right\|^{2} \\
& \leq \quad\left(1-\alpha_{n}\right)\left\|x_{n}-q\right\|^{2} \\
& \quad+\alpha_{n}\left\|\left(I-\lambda_{n} \mu F\right)\left(y_{n}-q\right)-\lambda_{n} \mu F(q)\right\|^{2} \\
& \leq \quad\left(1-\alpha_{n}\right)\left\|x_{n}-q\right\|^{2} \\
& \quad+\alpha_{n}\left\{\left(1-\lambda_{n} \tau\right)\left\|y_{n}-q\right\|^{2}\right. \\
& \left.\quad-2 \lambda_{n} \mu\left[\left\langle F(q), y_{n}-q\right\rangle-\lambda_{n} \mu\left\langle F(q), F\left(y_{n}\right)\right\rangle\right]\right\}
\end{aligned}
$$




$$
\begin{aligned}
\leq & \left(1-\alpha_{n}\right)\left\|x_{n}-q\right\|^{2}+\alpha_{n}\left(1-\lambda_{n} \tau\right)\left\|S_{n} x_{n}-q\right\|^{2} \\
& -2 \alpha_{n} \lambda_{n} \mu\left\langle F(q), y_{n}-q\right\rangle \\
& +2 \alpha_{n} \lambda_{n}^{2} \mu^{2}\|F(q)\|\left\|F\left(y_{n}\right)\right\| \\
\leq & \left(1-\alpha_{n} \lambda_{n} \tau\right)\left\|x_{n}-q\right\|^{2}-2 \alpha_{n} \lambda_{n} \mu\left\langle F(q), y_{n}-q\right\rangle \\
& +2 \alpha_{n} \lambda_{n}^{2} \mu^{2}\|F(q)\|\left\|F\left(y_{n}\right)\right\| \\
= & \left(1-\alpha_{n} \lambda_{n} \tau\right)\left\|x_{n}-q\right\|^{2} \\
& +\alpha_{n} \lambda_{n} \tau\left[\frac{2 \mu}{\tau}\left\langle F(q), q-y_{n}\right\rangle\right. \\
& \left.+2 \lambda_{n} \frac{\mu^{2}}{\tau}\|F(q)\|\left\|F\left(y_{n}\right)\right\|\right] .
\end{aligned}
$$

By virtue of Lemma 8 with (C1), (C2), and (40), we obtain that

$$
\lim _{n \rightarrow \infty}\left\|x_{n}-q\right\|=0
$$

that is, the sequence $\left\{x_{n}\right\}$ generated by (7) converges strongly to the unique element $q \in K$ of the variational inequality (1). This completes the proof.

Theorem 13. Let $H$ be a real Hilbert space and let $F: H \rightarrow H$ be a relaxed $(\gamma, r)$-cocoercive and L-Lipschitzian continuous mapping with $0<\mu<2\left(r-\gamma L^{2}\right) / L^{2}$. Let $\left\{T_{i}\right\}_{i=1}^{N}: H \rightarrow H$ be a finite family of nonexpansive mappings such that $K=$ $\bigcap_{i=1}^{N} \operatorname{Fix}\left(T_{i}\right) \neq \phi$. Define sequence $\left\{x_{n}\right\}$ by $x_{0} \in K$ in the following manner:

$$
\begin{aligned}
y_{n} & =\eta_{1}^{(n)} T_{1} x_{n}+\eta_{2}^{(n)} T_{2} x_{n}+\cdots+\eta_{N}^{(n)} T_{N} x_{n}, \\
x_{n+1} & =\left(1-\alpha_{n}\right) x_{n}+\alpha_{n}\left(I-\lambda_{n} \mu F\right) y_{n}, \quad n \geq 0,
\end{aligned}
$$

where $\left\{\alpha_{n}\right\} \subset(0,1)$ and $\left\{\eta_{i}^{(n)}\right\}_{i=1}^{N}$ are finite sequences of positive numbers such that $\sum_{i=1}^{N} \eta_{i}^{(n)}=1$ for all $n \geq 0$. If (C1), (C2), and the following control conditions are satisfied:

(i) $0<\liminf _{n \rightarrow \infty} \alpha_{n} \leq \limsup _{n \rightarrow \infty} \alpha_{n}<1$,

(ii) $\lim _{n \rightarrow \infty}\left|\eta_{i}^{(n+1)}-\eta_{i}^{(n)}\right|=0$,

then $\left\{x_{n}\right\}$ converges strongly to the unique element $q \in K$ of the variational inequality (1).

Proof. Clearly, a $k$-strict pseudocontraction reduces to a nonexpansive mapping as $k=0$. Putting $\beta=1$, iterative scheme (7) reduces to (43), and the desired conclusion follows immediately from Lemma 11 and Theorem 12 . This completes the proof.

Theorem 14. Let $H$ be a real Hilbert space and let $F: H \rightarrow$ $H$ be a r-strongly monotone and L-Lipschitzian continuous mapping with $0<\mu<2 r / L^{2}$. Let $T: H \rightarrow H$ be a $k$-strict pseudocontractions such that $\operatorname{Fix}(T) \neq \phi$. Define sequence $\left\{x_{n}\right\}$ by $x_{0} \in K$ in the following manner:

$$
\begin{gathered}
y_{n}=\left(1-\beta_{n}\right) x_{n}+\beta_{n} T x_{n}, \\
x_{n+1}=\left(1-\alpha_{n}\right) x_{n}+\alpha_{n}\left(I-\lambda_{n} \mu F\right) y_{n}, \quad n \geq 0,
\end{gathered}
$$

where $\left\{\lambda_{n}\right\},\left\{\alpha_{n}\right\}$, and $\left\{\beta_{n}\right\}$ are some positive sequences in $(0,1)$. If (C1), (C2), and the following control conditions are satisfied:

(i) $0<\liminf _{n \rightarrow \infty} \alpha_{n} \leq \limsup _{n \rightarrow \infty} \alpha_{n}<1$,

(ii) $\beta_{n} \in(0,1-k]$ and $\lim _{n \rightarrow \infty} \beta_{n}=\lambda$,

then $\left\{x_{n}\right\}$ converges strongly to the unique element $q \in \operatorname{Fix}(T)$ of the variational inequality (1).

Proof. By Remark 3, a relaxed $(\gamma, r)$-cocoercive mapping includes $r$-strongly monotonic mapping as a special case. Putting $N=1$, that is, $W_{n}=T$, iterative scheme (7) reduces to (44), and the desired conclusion follows immediately from Lemma 11 and Theorem 12. This completes the proof.

\section{Conflict of Interests}

The authors declare that there is no conflict of interests regarding the publication of this paper.

\section{Acknowledgments}

Supported by the National Science Foundation of China (11001287), Natural Science Foundation Project of Chongqing (CSTC, 2012jjA00039), and Science and Technology Research Project of Chongqing Municipal Education Commission (KJ130712, KJ130731).

\section{References}

[1] G. Stampacchia, "Formes bilineares coercitives sur les ensembles convexes," Comptes Rendus Hebdomadaires des Séances de l'Académie des Sciences, vol. 258, pp. 4413-4416, 1964.

[2] R. Glowinski, Numerical Methods for Nonlinear Variational Problems, Springer, New York, NY, USA, 1984.

[3] E. Zeidler, Nonlinear Functional Analysis and Its Applications, Springer, New York, NY, USA, 1985.

[4] H. K. Xu and T. H. Kim, "Convergence of hybrid steepestdescent methods for variational inequalities," Journal of Optimization Theory and Applications, vol. 119, no. 1, pp. 185-201, 2003.

[5] L. C. Zeng, Q. H. Ansari, and S. Y. Wu, "Strong convergence theorems of relaxed hybrid steepest-descent methods for variational inequalities," Taiwanese Journal of Mathematics, vol. 10, no. 1, pp. 13-29, 2006.

[6] G. Marino and H.-K. Xu, "A general iterative method for nonexpansive mappings in Hilbert spaces," Journal of Mathematical Analysis and Applications, vol. 318, no. 1, pp. 43-52, 2006.

[7] Y. Miao and J. Li, "Weak and strong convergence of an iterative method for nonexpansive mappings in Hilbert spaces," Applicable Analysis and Discrete Mathematics, vol. 2, no. 2, pp. 197-204, 2008. 
[8] P.-E. Maingé, "Extension of the hybrid steepest descent method to a class of variational inequalities and fixed point problems with nonself-mappings," Numerical Functional Analysis and Optimization, vol. 29, no. 7-8, pp. 820-834, 2008.

[9] D.-J. Wen, "Projection methods for a generalized system of nonconvex variational inequalities with different nonlinear operators," Nonlinear Analysis, Theory, Methods and Applications, vol. 73, no. 7, pp. 2292-2297, 2010.

[10] D.-J. Wen, X.-J. Long, and Q.-F. Gong, “Convergence analysis of projection methods for a new system of general nonconvex variational inequalities," Fixed Point Theory and Applications, vol. 2012, article 59, 2012.

[11] L. C. Zeng, N. C. Wong, and J. C. Yao, "Convergence analysis of modified hybrid steepest-descent methods with variable parameters for variational inequalities," Journal of Optimization Theory and Applications, vol. 132, no. 1, pp. 51-69, 2007.

[12] Y. Yao, M. A. Noor, R. Chen, and Y.-C. Liou, "Strong convergence of three-step relaxed hybrid steepest-descent methods for variational inequalities," Applied Mathematics and Computation, vol. 201, no. 1-2, pp. 175-183, 2008.

[13] N. Buong and L. T. Duong, "An explicit iterative algorithm for a class of variational inequalities in Hilbert spaces," Journal of Optimization Theory and Applications, vol. 151, no. 3, pp. 513524, 2011.

[14] J. S. Jung, "A general iterative scheme for $k$-strictly pseudocontractive mappings and optimization problems," Applied Mathematics and Computation, vol. 217, no. 12, pp. 5581-5588, 2011.

[15] Q. F. Gong and D. J. Wen, "General convergence analysis of projection methods for a system of variational inequality in $q$ uniformly smooth Banach spaces," Journal of Inequalities and Applications, vol. 2013, article 481, 2013.

[16] F. E. Browder, "Convergence of approximants to fixed points of nonexpansive nonlinear mappings in Banach spaces," Archive for Rational Mechanics and Analysis, vol. 24, no. 1, pp. 82-90, 1967.

[17] F. E. Browder and W. V. Petryshyn, "Construction of fixed points of nonlinear mappings in Hilbert space," Journal of Mathematical Analysis and Applications, vol. 20, no. 2, pp. 197228, 1967.

[18] K. Aoyama, H. Iiduka, and W. Takahashi, "Weak convergence of an iterative sequence for accretive operators in Banach spaces," Fixed Point Theory and Applications, vol. 2006, article 35390, 2006.

[19] H. K. Xu, "An iterative approach to quadratic optimization," Journal of Optimization Theory and Applications, vol. 116, no. 3, pp. 659-678, 2003.

[20] X. Liu and Y. Cui, "The common minimal-norm fixed point of a finite family of nonexpansive mappings," Nonlinear Analysis, Theory, Methods and Applications, vol. 73, no. 1, pp. 76-83, 2010.

[21] H. Zhou, "Convergence theorems of fixed points for $k$-strict pseudo-contractions in Hilbert spaces," Nonlinear Analysis, Theory, Methods and Applications, vol. 69, no. 2, pp. 456-462, 2008.

[22] G. Marino and H.-K. Xu, "Weak and strong convergence theorems for strict pseudo-contractions in Hilbert spaces," Journal of Mathematical Analysis and Applications, vol. 329, no. 1, pp. 336-346, 2007.

[23] T. Suzuki, "Strong convergence of approximated sequences for nonexpansive mappings in Banach spaces," Proceedings of the American Mathematical Society, vol. 135, pp. 99-106, 2007.
[24] K. Goebel and W. A. Kirk, Topics in Metric Fixed Point Theory, vol. 28 of Cambridge Studies in Advanced Mathematics, Cambridge University Press, Cambridge, UK, 1990.

[25] G. L. Acedo and H.-K. Xu, "Iterative methods for strict pseudocontractions in Hilbert spaces," Nonlinear Analysis, Theory, Methods and Applications, vol. 67, no. 7, pp. 2258-2271, 2007. 


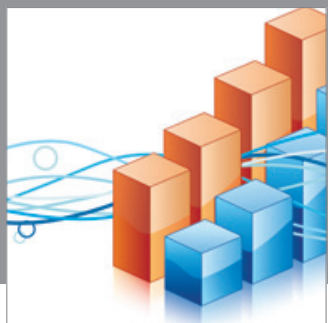

Advances in

Operations Research

mansans

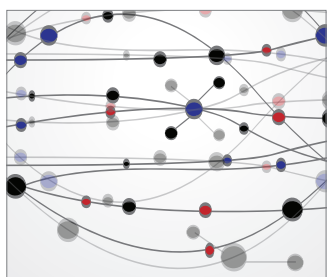

The Scientific World Journal
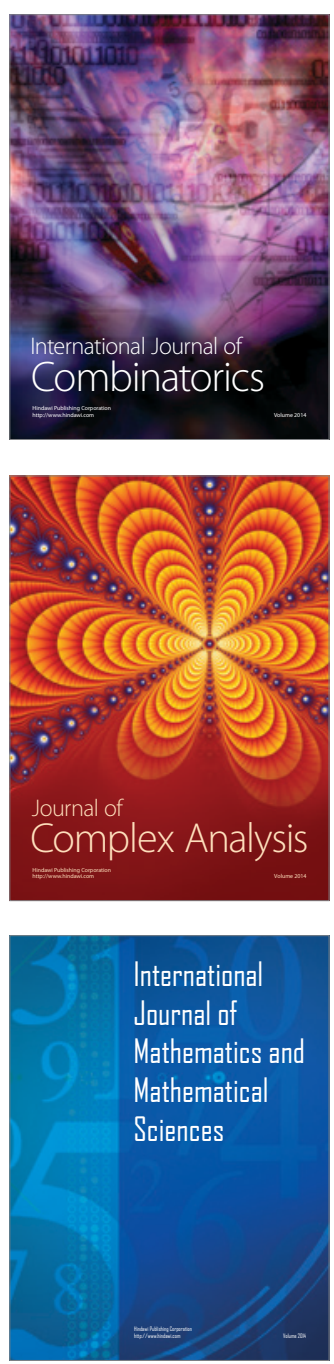
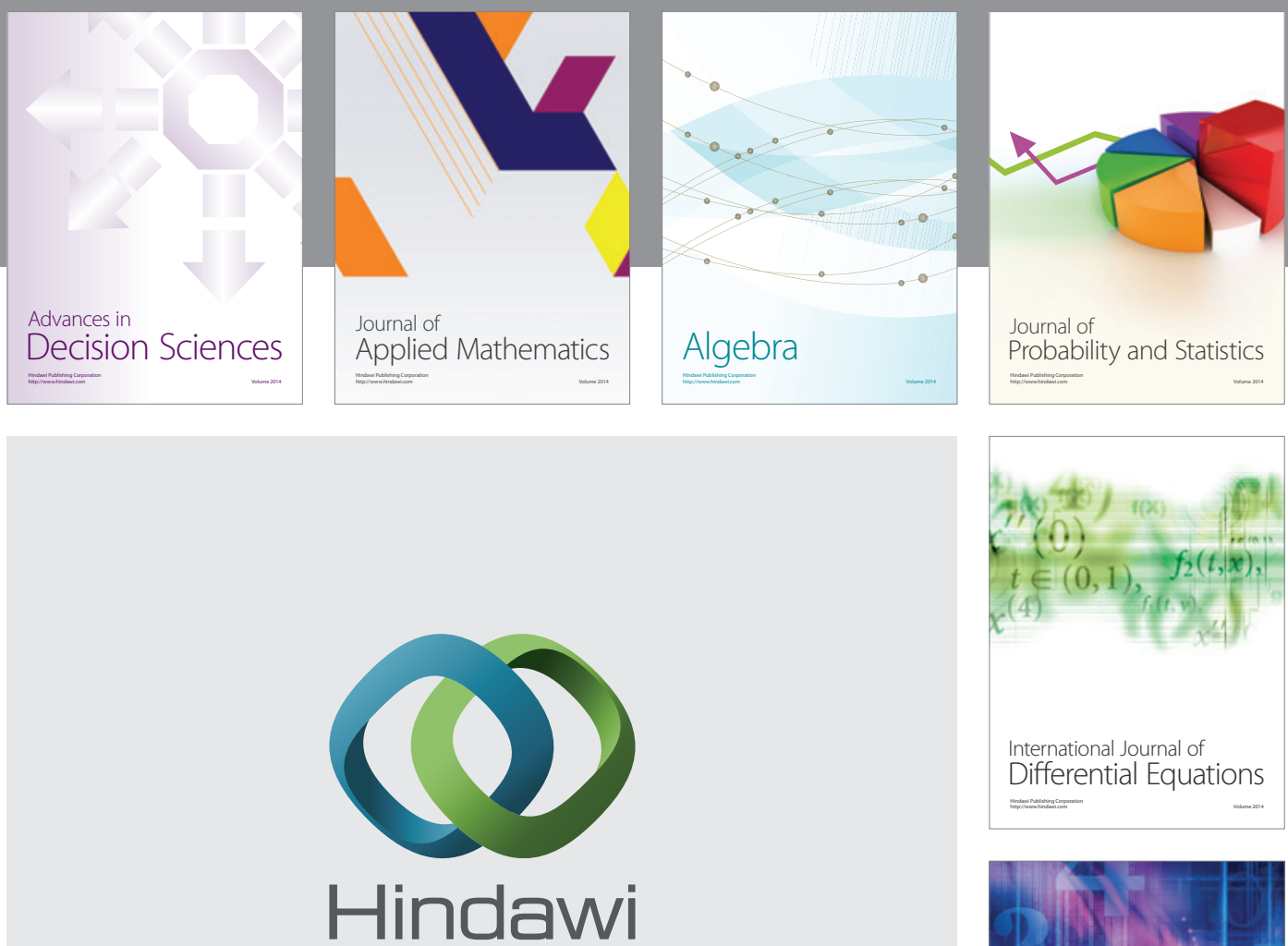

Submit your manuscripts at http://www.hindawi.com
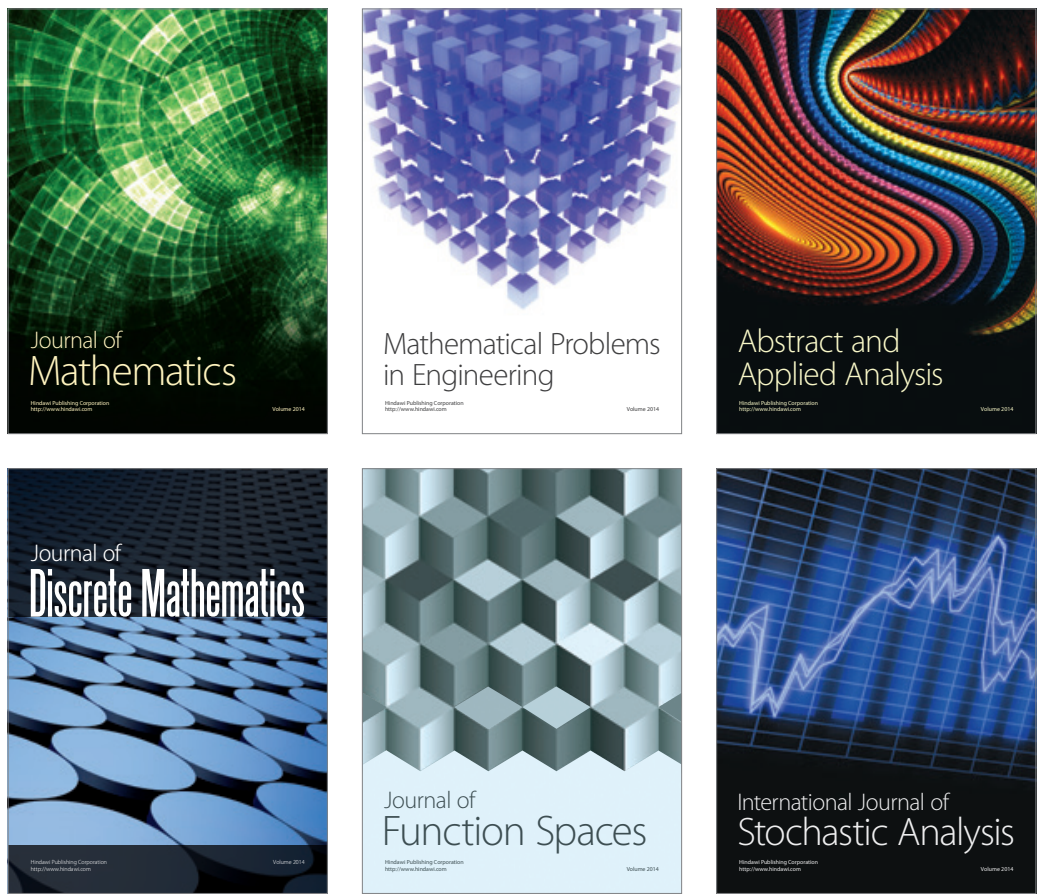

Journal of

Function Spaces

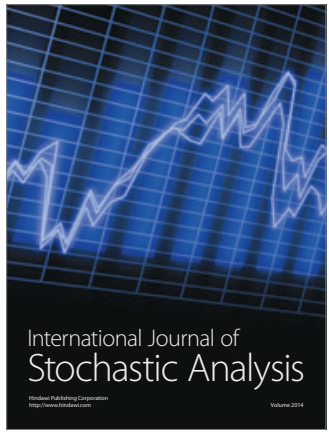

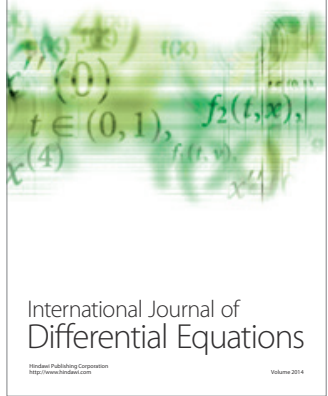
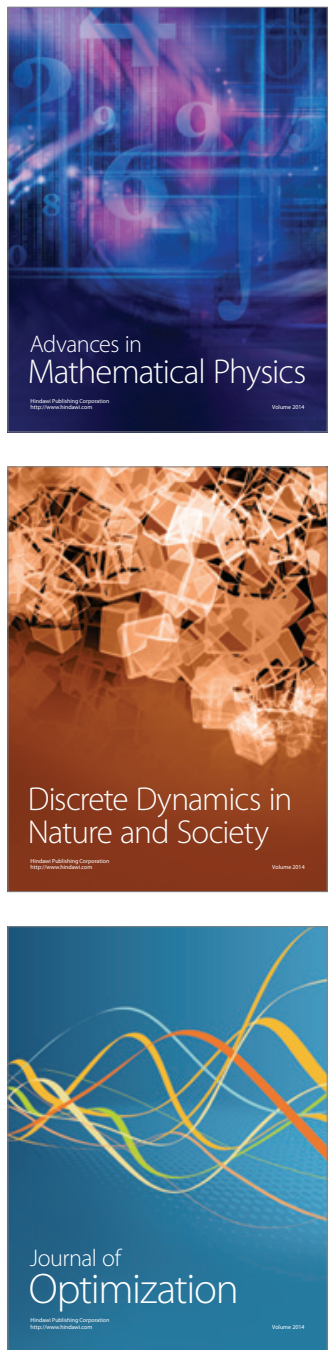